\title{
Missing Mass and Galactic Dust with a Size Greater than 200 Microns, Minimum Size of the Micrometric Dust around the Sun
}

\author{
Marc Mignonat \\ Société d'Astronomie des Pyrénées Occidentales, Pau, France \\ Email: mmignonat@libertysurf.fr
}

How to cite this paper: Mignonat, $M$. (2019) Missing Mass and Galactic Dust with a Size Greater than 200 Microns, Minimum Size of the Micrometric Dust around the Sun. Journal of Modern Physics, 10, 548-556.

https://doi.org/10.4236/jmp.2019.105038

Received: March 25, 2019

Accepted: April 21, 2019

Published: April 24, 2019

Copyright (c) 2019 by author(s) and Scientific Research Publishing Inc. This work is licensed under the Creative Commons Attribution International License (CC BY 4.0).

http://creativecommons.org/licenses/by/4.0/

\begin{abstract}
The studies of the glaciologists show that, since 30,000 years, the Earth receives every year approximately 40,000 tons of dust, dust with an average size about 200 microns. By determining of which volume these 40,000 T come and by showing that the density of this volume is significant within the density of the milky way, I have tried to estimate the mass of dust contained in the Galaxy. To support that this density close to earth is representative, arguments are given: 1) the distribution of great dust is largely homogeneous in the galaxy (what does not exclude the existence of gas or dust clouds with different densities in the milky way); 2) there would be a minimum size that I have calculated for micrometeorites in the solar environment, and so there would be a lack of the micrometeorites with a size between 5 and 50 microns. So the density would not be greater in the solar system. Next, a very simple rough calculation (as the one made by the observatory of Paris in 1910) allows estimating this mass near 4 times that of the dark matter. So, the interstellar dust with a large size $(>200 \mu)$ could it be the missing mass? A verification method is proposed to confirm or refute this hypothesis.
\end{abstract}

\section{Keywords}

Dark Matter, Dust, Galaxies: ISM

\section{Introduction}

Every year, the Earth receives 40,000 $\mathrm{T}$ of dust and this figure has not varied since at least 30,000 years [1]. A study, in 2016 [2] of the marine sediments shows there is not variation of this value since about 40 million years with a maximum at the end of Eocene ( $35 \mathrm{Myr}$ ) and at the end of Miocene (8.2 Myr). 
In her thesis of 2010, Dobriča [3] specifies that on every $\mathrm{m}^{2}$, it falls an average of one micrometeorite every year (or 30.000 tons against only 10 tons of macroscopic meteorites). They are too small so that we notice it (their size is about 200 microns). For example, during a collection in Antarctica in 2006, in $24 \mathrm{~m}^{3}$ of the filtered snow were extracted more than 1300 micrometeorites. This average size of 200 microns does not correspond to what we suppose on the dark matter.

The dark matter is a category of hypothetical matter, used to explain astrophysical observations where there is a missing mass, (for instance the element abundances predicted by nucleosynthesis, the detailed form of anisotropies in the cosmic microwave background particularly at redshift 1000, the estimations of mass of the galaxies and of the clusters of galaxies to promote the initial structure formation). But we can note that, in all these "particulate" or "gaseous" observations, we do not find measures about dust greater than the micron.

Various hypotheses are explored on the composition of dark matter: molecular gas, dead stars, brown dwarfs in large numbers, black holes, etc. As we do not find enough visible matter, astrophysicists suppose a non baryonic matter with other particles, (e.g. neutrinos, axions, perhaps superpartners such as the neutralino). These other particles are grouped in the generic name of "WIMP".

The MOND theory is an alternative recipe for the missing mass [4]. This theory says that this dark matter would not exist, and makes the hypothesis that its existence would be due to a partial ignorance of laws of gravity.

Astrophysicists like Magnan, [5] consider that dark matter does not exist, that it is an error resulting from a too big uncertainty which exists on the measures of speeds and masses within the galaxies.

So, I had the curiosity to try to estimate the global mass of the dust in the Galaxy with the approach used by the observatory of Paris in 1910 and compare it with the mass of the dark matter. But first, we have to know if the density in the solar environment would be representative of that of the Galaxy. For that, we have to question the homogeneous distribution of dust and see if the size of dust would be representative.

\section{Dust Distribution in the Galaxy and Size of Dust}

\subsection{Global Homogeneity in the Galaxy}

1) The calculation, resulting from the observation (estimation in 1910 of 10,000 T/year on the Earth [6]), gives an absorption of 2 magnitude by Kpc (what means that a star situated near the galactic center in $8.5 \mathrm{Kpc}$ undergoes an extinction about 16 magnitudes and thus is often invisible). This extinction, proportional to the distance, involves a homogeneous distribution of the dusts between us and the galactic center.

2) We can cite the thesis of e.g. Arab [7] about the evolution of interstellar dust. In this exhaustive thesis, he considers that the interstellar medium is constituted by all the matter filling the space between the stars. This matter is made of an extremely tenuous mixture of gas and dust of an atom $/ \mathrm{cm}^{3}$. The dusts are 
solid particles with a size between the nanometer and the micron. Their small size near the wavelengths of emission $(<100 \mathrm{~nm})$ explains their hotter temperatures or around $17 \mathrm{k}$ or around $30 \mathrm{k}$. There is a homogeneous distribution but the dusts with a size greater than the micron are not considered.

3) Zagury [8] already finds incompatibilities between the standard theory of the interstellar extinction and the observations. With the observations resulting from the International Ultraviolet satellite, the extinction in the UV spectrum with the $2200 \AA$ bump, .was studied for the nebulae, and in directions of low and very low reddening. It is found that extinction occurs linear function from the near IR to the extreme UV, what is incompatible with the standard theory of the interstellar extinction. He notes that the observation of the reddened light from the stars, is contaminated by the diffused light with a very low angle of diffusion. He says this questions all the models of dust actually used since the consequence is that there are no differences in average properties of the interstellar dust in different directions. It is always written that "the interstellar dust represents a very low part of the interstellar matter, essentially gaseous". The quantity of dust is constant, most probably because the distribution of the dust is at first glance, homogeneous within the Galaxy.

4) Studies [9] [10] show an agitation of the dust and suggest a fragmentation of the clouds of gas: on a line of sight, it is noticed that the intensity of the absorption lines of $\mathrm{H}_{2} \mathrm{CO}$ varies from one year to the next (the recording was made during 18 years). It suggests turbulent movements of the interstellar matter which cause a slow renewal on the line of sight which the authors attribute to the movement of the earth around the sun and to the movement of the sun in the Galaxy $\left(20 \mathrm{~km} \cdot \mathrm{s}^{-1}\right.$ in the direction defined by $\alpha \approx 18 \mathrm{~h}$ and $\left.\delta \approx 30^{\circ}\right)$. But even if the gaseous concentrations are variable, for the authors, "It seems thus finally that in this type of cloud, the dusts are distributed in a relatively uniform way and that there are no strong fluctuations".

\subsection{Size of the Dust}

1) The old studies of the 1950s, for the particles "very large relative to the wavelength", determined from the measures of the color indices [11], and from the ratios of the absorption coefficients that there was no place for neutral absorption in the milky way [12]. However, there were few isolated studies as that of Alter [13] which, depending on the galactic longitude, found a larger coefficient of $0.83 \mathrm{mag} / \mathrm{Kpc}$ (instead of 0.17 ), which required a much higher mass of dust. The conclusion by Delay in 1954 [14] of these studies is that, from the study of the only absorption it is impossible to deduce what is the nature of the particles responsible for the absorption.

2) It is written in 1946 that the dust cannot exceed a few thousand of angstrom [15]. Gyngard [16] finds 25 microns in 2018 or Gregory in 2005 [17] gives an approximate mass of $10^{-17} \mathrm{~kg}$. The reason of the small size of the particles is explained by a balance between the growth of grains possible for every atom except $\mathrm{H}$ and $\mathrm{He}$ when the temperature is greater than $10-20^{\circ} \mathrm{K}$ and the 
destruction by shocks or evaporation [15].

3) Also old studies show that the radiation pressure $\operatorname{Pr}$ on particles much larger than the wavelength prevent the presence in space of particles of few tens of microns. The particles are in balance between the radiation pressure $\mathrm{Pr}$ and the gravitational force Fg; these two forces diminish with the square of the distance, so the ratio between Pr and Fg is constant [14]; when Pr overcomes, the grains are pushed back to several parsecs from the star [18].

4) It is, however, interesting to determine the minimum radius $r$ of grains that will not be pushed back and will remain in the solar environment. A simple calculation (see calculation in Appendix) allows setting the minimum radius $r$ to $r=3 \sigma T^{4} R_{0} / 8 G M_{0} \rho$ (with $\sigma$ constant of Stefan, $T$ temperature of the star, $R o$ radius of the star with a mass $M o, G$ constant of gravitation and $\rho$ density of the grain); for the sun, $r \approx 120 \rho$ (so a radius of $120 \mu$ or a size around $240 \mu$ for an object with density 1 , a size of $64 \mu$ for a chondrite with a density 3.8 or $50 \mu$ for a siderite with density 4.8 ). This minimum size is in conformity with the samples taken by Dobrica in Antarctica(3), as well as the latest studies from the Cassini spacecraft with the collection of 36 micrometeorites from outside the solar system [19] and from the collection of dust in 2017 on the rooftops of several European cities [20].

\subsection{Finally about Homogeneity and Size}

From these studies cited above in paragraphs $2-1$ and 2-2, it appears: first, it is reasonable to believe that the distribution of the dust is globally homogeneous (what does not exclude the existence of gas or dust clouds) and that the observed rate of dust impinging the Earth is representative of the Galaxy. We can note that the recent hypotheses made only from the studies upon the gases (where we do not find dust greater than the micron) cannot explain the 40,000 $\mathrm{T}$ annually falling on the earth.

Second, there would be no a highter dust density around the sun since a category for dust is under-represented. The old studies show the difficulty in studying the neutral dust and determine for the small grains a maximum size near the wavelength. It is important to note that a minimum size of micrometeorites can also be calculated in the terrestrial environment. So, the particles of an approximate size between 6 and $50 \mu$ would be under-represented. More specifically, taking into account the increasing density, there would be a gradual decrease of the quantity of dust from $240 \mu$ to arrive at a near absence to a size of $50 \mu$ (since only the particles with a density greater than 5 could be smaller). It is thus assumed that these particles of intermediate size would be more abundant at equidistance from the stars.

\section{Approximate Calculation of the Mass of Dust of the Galaxy}

\subsection{At First, the Volume of the Galaxy Is to Determine}

In the usual way for our Galaxy, its form can be approximated by an ellipsoid of 
revolution whose the equatorial radius is $R^{\prime}=50,000$ ly and the polar radius $R^{\prime \prime}$ $=5000$ ly. So, we obtain the volume $V g$ by multiplying twice the equatorial radius $R^{\prime}$ by the polar radius $R^{\prime \prime}$.

$$
V g=(4 / 3) \pi R^{\prime 2} \times R^{\prime \prime}=5 \times 10^{61} \mathrm{~m}^{3}
$$

\subsection{Secondly, It Is Necessary to Determine the Volumetric Concentration of the Dust of the Galaxy}

For calculating this value, we have to estimate from which volume come the 40,000 T of dust, every year received by the earth.

The earth in one year, is going to sweep the volume of a torus whose the large radius $R$ is the radius of the earth orbit and the small radius $r$ of the torus is the radius of the sphere in which the attraction of the earth is applied. This radius $r$ is difficult to estimate.

If we are considering that the dusts are in solar orbit, then it is necessary to take the maximal radius of gravitational influence, with the points of Lagrange L1 and L2 situated in approximately $0.01 \mathrm{UA}=1.5$ millions $\mathrm{km}$. But, this maximalist hypothesis can be ruled out because then, year after year, the Earth passing approximately in the same place, the collected quantity of dust would had decreased and approached zero.

A possible calculation to have the largest volume, is to consider that the component of the speed of the dust is zero in the plan of the ecliptic; and to take as maximal distance the time to go to the Earth during its time of passage. At a given point, we can supposed the earth have a maximal influence during its time of passage $\mathrm{Tp} . \mathrm{Tp}=$ diameter $/$ speed $\approx 12,000 / 30=400 \mathrm{~s}$. This influence being inversely proportional to the square of the distance, we are going to avoid an integral calculation by making the approximation that the time of influence $t$ will be multiplied by two, so $t=400 \mathrm{~s} \times 2=800 \mathrm{~s}$.

The concerned dust will go to the Earth during this journey of 800 s. Even by supposing the acceleration $g$ of the gravity equal to that is to the surface of the earth, so $\mathrm{g}=10 \mathrm{~m} \cdot \mathrm{s}^{-2}$ what is excessive, we arrive only at a traveled distance $\mathrm{d}$ of $3200 \mathrm{~km}\left(\mathrm{~d}=\mathrm{gt}^{2} / 2\right)$.

Then, we shall have, in priori a surface of sweeping in a high range by supposing a small radius of the torus of $9500 \mathrm{~km}$ swept by the earth. (A similar calculation had already been made in 1911 by Salet [6] of the Observatory of Paris who, trying to calculate the absorption from the quantity of dusts annually received by the earth (estimation in 1910 of 10,000 T every year), had made an estimation of the volume of space of which came these dust. He estimated the earth received the dust in a radius $\mathrm{x} / \mathrm{x}^{2}=1.2 \rho^{2}$ with $\rho$ the radius of the earth and he multiplied by 5 the amount of dust to take into account the attraction of the sun. So, he had found an equivalent radius of $11,914 \mathrm{~km}$ what is lightly superior to the value I find, but in 1910, the galactic movement was not known and the sun was supposed motionless.

The volume of the torus $V t$ in which the dusts are collected by the earth is: 


$$
V t=2 \pi r 2 R=2 \pi 2\left(9.5 \times 10^{6}\right) 2 \times 1.5 \times 10^{11} \approx 2.7 \times 10^{26} \mathrm{~m}^{3} .
$$

So, the volumetric concentration $\rho g$ of the dusts in the space is:

$$
\rho g=4 \times 10^{7} / 2.7 \times 10^{26} \approx 1.5 \times 10^{-19} \mathrm{~kg} \cdot \mathrm{m}^{-3}
$$

This value is very high (we usually count for the interstellar space an atom by $\mathrm{cm}^{3}$ or approximately $\left(1.67 \times 10^{-21} \mathrm{kgm}^{-3}\right)$.

\subsection{The Total Mass Mt of the Dust in the Galaxy Can Be Estimated}

$$
(1)(2) \geq M t=V g \times \rho g \approx 7.5 \times 10^{42} \mathrm{~kg}
$$

The visible mass of the Galaxy is considered near $4 \times 10^{41} \mathrm{~kg}$ [21] [22].

So, that of the dark matter, nearly 5 times larger, would be $2 \times 10^{42} \mathrm{~kg}$, i.e. approximately 4 times smaller than the value that I have just calculated. However this value has the same order of magnitude.

\section{Hypotheses and Conclusion}

The orders of magnitude, obtained by the calculations that have just been made, invite reflection. They induce me to consider that the missing mass could be found with a much more elaborate classical gravitational calculation than the one I have just outlined, particularly with the inclusion of the detailed structure of the Galaxy, an improvement by the glaciologists or geologists of the calculation of the dust amount annually received by the Earth and the measurement in the space of the quantity of dust with a size greater than micrometer.

I am not the only one to question about the dark matter. But, perhaps, it is the first study which estimates the galactic dust mass from the dust $(>200 \mu)$ received by the Earth and in this study, I am assuming the distribution of dust in the Galaxy is globally homogeneous (what does not exclude local variations with great clouds of gas or dust). This global homogeneity, even if I have cited several studies in its favor, questions the standard theory of the interstellar extinction, so, we have to be prudent. Other reason is that I am supposing the dust comes from the outside of the solar system because the quantity of dust is constant since 40 million years, but 40 million years is not a long time on the astronomical scale.

We can note that the calculation allows finding that the mass would exceed the known values of the missing mass by at least four orders of magnitude. So, to explain this excess of matter, we have to suppose that, either we are by chance in an area of the Galaxy with a high density (it is unlikely), or the dust is mainly in the interplanetary zone (this area could go very far beyond the Oort cloud). It is then logical to think that this dust exists around each star. By multiplying this amount of interplanetary dust by the number of stars, we would then find more precisely the missing mass. This hypothesis has the advantage of preserving the mechanisms already known from the observations on galaxies and their formation. 
A point for a hypothetical origin from the outside of the solar system is that the IR radiation of the dusts is larger in the galactic plan and no in the ecliptic plan (and so we could assume the maximum of micrometric dust is not in the ecliptic plan).

But the best verification about these hypotheses could be given by the collection directly in the space and, so by the measurement of the density of the dust inside the solar system. The minimal radius $r$ of the dust in the solar environment would be around $r \approx 120 \mu / \rho$ ( $\rho=$ density of the micrometeorite).

And, if the dust is interplanetary, the density would be higher in the ecliptic plan; if the dust is coming from a region more distant from the solar system, so the density of the micrometric dust inside various points of the solar system (e.g. inside and outside of the ecliptic plane) would be approximately constant.

\section{Conflicts of Interest}

The author declares no conflicts of interest regarding the publication of this paper.

\section{References}

[1] Winckler, G. and Fischer, H. (2006) Science, 313, 491. https://doi.org/10.1126/science.1127469

[2] Chavrit, D., Moreira, M. and Moynier, F. (2016) Earth and Planetary Science Letters, 436, 10-18.

[3] Dobriča, E. (2010) Micrométéorites concordia: Des neiges antartiques aux glaces cométaires. Thèse Paris 11 en partenariat avec Université Paris-Sud. Faculté des Sciences d'Orsay.

[4] Tortora, C., Jetzer, P. and Napolitano, N.R. (2012) Dark Matter and Alternative Recipes for the Missing Mass.

[5] Magnan, C. (2014) Le théorème du jardin.

[6] Salet, P. (1911) Bulletin Astronomique, 28, 241-251.

[7] Arab, H. (2012) Evolutions des poussière interstellaires: Apport des données de l'observatoire spatial Herschel. Thèse, Université Paris Sud., Paris, XI.

[8] Zagury, F. (2000) New Astronomy, 5, 285-298.

[9] Boissé, P. and Thoraval, S. (1990) La distribution des poussières à l'échelle de l'unité astronomique dans les nuages interstellaires. A and A 228, 483.

[10] Marscher and Als (1993) Astrophysical Journal, 419, L101. https://doi.org/10.1086/187147

[11] Stebbens, J. and Whitford, A.E. (1936) Astrophysical Journal, 84, 132. https://doi.org/10.1086/143755

[12] Greenstein, J.L. and Henyey, I.G. (1939) Astrophysical Journal, 89, 647. https://doi.org/10.1086/144088

[13] Alter, G. (1949) Memoirs and Observ. Czechoslovak astr. Soc., Prague, No. 10.

[14] Dufay, J. (1954) Nébuleuses galactiques et matière interstellaire. Albin Michel Ed., Paris.

[15] Oort, J.H. and Van de Hulst, H.C. (1946) Bulletin of the Astronomical Institutes of the Netherlands, 10, 187.

[16] Gyngard, F., Jadhav, M., Nittler, L.R., Stroud, R.M. and Zinner, E. (2018) Geochi- 
mica et Cosmochimica Acta, 221, ॠ60-86.

[17] Gregory, L. and Matloff, L.J. (2005) Applications of the Electrodynamic Tether to Iinterstellar Travel.

[18] Schalén, C. (1929) Astronomische Nachrichten, 236, 249. https://doi.org/10.1002/asna.19292361602

[19] Altobelli, N. and Als (2016) Science, 352, 312-318. https://doi.org/10.1126/science.aac6397

[20] Genge, M.J., Larsen, J., Van Ginneken, M. and Suttle, M.D. (2017) Geology, 45, 119-122. https://doi.org/10.1130/G38352.1

[21] Andreas, Küpper, H.W., Balbinot, E., Bonaca, A., Johnston, K.V., Hogg, D.W., Kroupa, P. and Santiago, B.X. (2015) Astrophysical Journal, 803, 80. https://doi.org/10.1088/0004-637X/803/2/80

[22] McMillan, P.J. (2011) Monthly Notices of the Royal Astronomical Society, 414, 2446-2457. https://doi.org/10.1111/j.1365-2966.2011.18564.x 


\section{Appendix: Calculation of the Minimal Size of the Dust around a Star}

We have a body A with a size much larger than the wavelengths of the electromagnetic waves emitted by the star (otherwise the calculations are not valid). The movement of this body A will be linked to the balance between the attraction force $F g$ of the star and the force Fr of the radiation flow from the star. The force $F r$ will be proportional to the surface of the body A exposed to the flow and will also depend on the albedo of A. We simplify the problem by considering that the body A is spherical with an average radius $r$ and we do not consider the albedo. So, the surface of A which receive the energy is a hemisphere with a surface $\pi r^{2} / 2$. The received energy $E r$ by the body A is:

$E_{r}=\left(F_{0} R_{0}^{2} / R^{2}\right)\left(\pi r^{2} / 2\right) .\left(F_{0} R_{0}^{2} / R^{2}\right)$ is the stellar flux at the la distance $R$ between the star and the body A (Fo: stellar flux at the stellar radius $R o$ ). The gravitational energy $E_{g}$ is: $E_{g}=(G M / R)\left(4 \pi r^{3} \rho / 3\right)$ with M mass of the star, $G$ constant of gravitation, $\rho$ density of the body A. By equalizing $E_{r}$ and $E_{g}$ and by posing Fo $=\sigma T^{4}$, we obtain: $r=3 \sigma T^{4} R_{0}^{2} / 8 G M R \rho$ (3) with $T$ temperature of the star, Ro radius of the star, $\sigma=$ cte of Stefan. We want to calculate the minimum radius $r$ that allows the movement of an object towards the star. In this case $R=R o$, and so (3) becomes $r=3 \sigma T^{4} R_{0} / 8 G M \rho$ (4) For the sun (4) can be written $r \approx 1.26 \times 10^{-4} / \rho$ meters or $r \approx 0.12 \mathrm{~mm} / \rho$ (with $T=5778 \mathrm{~K}$, $\left.R o=695.5 \times 10^{6} \mathrm{~m}, M=1.99 \times 10^{30} \mathrm{~kg}\right)$. 